\title{
A New-Type Equilibrium of Flowing Plasma Dominated by the Hall Effect
}

\author{
Jun-ya SHIRAISHI, Masaru FURUKAWA and Zensho YOSHIDA \\ Graduate School of Frontier Sciences, The University of Tokyo, Kashiwa 277-8561, Japan
}

(Received 15 July 2006 / Accepted 22 September 2006)

\begin{abstract}
New type of axisymmetric magnetohydrodynamic (MHD) equilibrium of toroidally flowing plasma, which cannot be found in the framework of the ideal MHD, emerges due to the Hall and toroidal effects. If either of these effects is neglected, only a conventional MHD-type equilibrium is obtained. Numerical solutions of both types are calculated in toroidal geometries to simulate the equilibrium states in the Ring Trap-1 device constructed at the University of Tokyo. Numerical analysis shows that when the toroidal flow is increased, the plasma shifts inward in the new-type equilibrium, while in the MHD type the outward shift occurs due to the centrifugal effect.
\end{abstract}

(C) 2006 The Japan Society of Plasma Science and Nuclear Fusion Research

Keywords: flowing plasma, axisymmetric MHD equilibrium, toroidal flow, Hall effect, internal conductor device, dipole magnetic field

DOI: $10.1585 /$ pfr. 1.050

\section{Introduction}

The Ring Trap-1 (RT-1) device [1] is a toroidal system equipped with a levitated internal ring creating a magnetosphere-like configuration. This system can produce a variety of magnetic structures by using external coils, e.g., a vertical field coil. Such configurations have been applied to fundamental research in non-neutral plasma physics, such as the verification of stable confinement [2] and the driving of a high-speed toroidal flow [3]. As well, the system can be used to study the confinement of high-beta plasma by a planet's dipole field, as is observed in Jupiter's magnetosphere [4], which is being investigated in the Levitated Dipole eXperiment (LDX) project [5].

The experiment involving the RT-1 device concerns mainly the phenomena induced by plasma flows. Especially, it aims to examine the theory of self-organized states of flowing plasmas [6,7], which is called the "double Beltrami equilibrium" that predicts that the dynamical pressure of the flow balances the thermal pressure, yielding a high-beta plasma. Also, the theory indicates that the coupling of the flow with the Hall effect leads to equilibrium structures far richer than the ideal magnetohydrodynamic (MHD) ones, and in the RT-1, the plasma parameters are selected such that the Hall effect becomes prominent (see Sec. 4). However, the equilibria including the flow and the Hall effect have been analytically studied only in relatively simple geometries, such as cylindrical [6] or slab geometry [8].

In this paper, we investigate the coupling of the flow with the Hall effect in "toroidal" geometry. Since in the RT-1 device it is planned that a high-speed toroidal flow be driven in the order of Alfvén speed, we analyze the axisymmetric MHD equilibrium with pure toroidal flows.

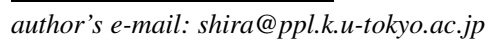

The analytical results show that the coexistence of the Hall effect and toroidicity produces a new-type equilibrium whose profile is drastically different from that of the ideal MHD type. Important issues to be addressed here are the significantly different profiles of the toroidal flow and density between the two types of equilibria, implying that their stability and confinement properties are totally different.

To analyze the equilibria in the RT-1 device, we calculate numerical solutions to the generalized Grad-Shafranov (GS) equations of both types in toroidal geometries. In the MHD-type equilibrium, as the volume-averaged toroidal flow increases, the peaks of the density and local beta value shift outward. In contrast, in the new-type equilibrium, the increased flow shifts the peaks inward.

We briefly review the related theories. It has been shown in Ref. [9] that the ideal axisymmetric MHD equilibrium including toroidal flow in a toroidal geometry is described by the generalized GS equation, which is a regular elliptic partial differential equation, and that numerical solutions can be obtained. Also, assuming that the solutions are in separable forms, the analytic solutions including toroidal flows in dipolar magnetic configurations have been obtained in Refs. [10,11] for the analysis of equilibria in the LDX. However, none of these solutions take into account coupling with the Hall effect.

This paper is organized as follows. In Sec. 2, axisymmetric Hall MHD equilibria with toroidal flows are introduced, and the significance of the toroidal effect is examined. In Sec. 3, it is shown that a new type of equilibrium emerges due to the Hall effect, and that the MHDtype equilibrium also exists. Numerical analyses of the two types of equilibria, which are applied to the RT-1 device, are described in Sec. 4 . Section 5 provides a summary of the results. 


\section{Basic Equations}

\subsection{Hall MHD equilibrium}

Let us consider the Hall MHD equilibrium. The governing equations are

$$
\begin{aligned}
& \nabla \cdot(n \boldsymbol{V})=0, \\
& n(\boldsymbol{V} \cdot \nabla) \boldsymbol{V}=(\nabla \times \boldsymbol{B}) \times \boldsymbol{B}-\nabla p, \\
& \nabla \times\left[\left(\boldsymbol{V}-\frac{\varepsilon}{n} \nabla \times \boldsymbol{B}\right) \times \boldsymbol{B}\right]=0, \\
& \nabla \cdot \boldsymbol{B}=0,
\end{aligned}
$$

where $n, \boldsymbol{B}, \boldsymbol{V}$, and $p$ are the number density, the magnetic field, the flow velocity, and the pressure, respectively. We have normalized the length by the system size $L_{0}, n$ by the representative number density $n_{0}, \boldsymbol{B}$ by the representative magnetic field $B_{0}, \boldsymbol{V}$ by the Alfvén speed $V_{\mathrm{A}}=B_{0} / \sqrt{\mu_{0} M n_{0}}\left(\mu_{0}\right.$ is the vacuum permeability and $M$ is the proton mass), and $p$ by $B_{0}^{2} / \mu_{0}$. The scaling coefficient $\varepsilon=\delta_{\mathrm{i}} / L_{0}$ measures the ion skin depth $\delta_{\mathrm{i}}=c / \omega_{\mathrm{pi}}=$ $\sqrt{M /\left(\mu_{0} n_{0} e^{2}\right)}(e$ is the elementary charge). Note that we need the equation of state to close the system. The only addition to the ideal MHD is the Hall term in (3), thus putting $\varepsilon=0$ recovers the ideal MHD equilibrium.

\subsection{Hall MHD equilibrium in a toroidal geometry}

We consider an axisymmetric toroidal geometry, then (4) indicates

$$
\boldsymbol{B}(R, Z)=\nabla \psi(R, Z) \times \nabla \phi+R B_{\phi}(R, Z) \nabla \phi,
$$

where $\psi\left(B_{\phi}\right)$ is the poloidal flux (toroidal component) of the magnetic field, and $R-\phi-Z$ are the standard cylindrical coordinates. The assumption of pure toroidal flow leads to

$$
V(R, Z)=R V_{\phi}(R, Z) \nabla \phi,
$$

where $V_{\phi}$ is the toroidal component of the flow velocity. Equation (1) is automatically satisfied. Substituting (5) and (6) into (2) yields

$$
\begin{aligned}
-\frac{n V_{\phi}^{2}}{R} \nabla R= & -\frac{\Delta^{*} \psi}{R^{2}} \nabla \psi+\left\{\psi, R B_{\phi}\right\} \nabla \phi \\
& -\frac{B_{\phi}}{R} \nabla\left(R B_{\phi}\right)-\nabla p,
\end{aligned}
$$

where $\Delta^{*} u=R \partial_{R}\left(R^{-1} \partial_{R} u\right)+\partial_{Z}^{2} u$ is the GS operator, and $\{P, Q\}=-(\nabla P \times \nabla Q) \cdot \nabla \phi=$ $-R^{-1}\left[\left(\partial_{Z} P\right)\left(\partial_{R} Q\right)-\left(\partial_{R} P\right)\left(\partial_{Z} Q\right)\right]$ is the Poisson bracket. From the toroidal component of (7), we get the first surface quantity,

$$
R B_{\phi}=I(\psi) .
$$

Then (3) and (7) reduce to

$$
\begin{aligned}
& \frac{\varepsilon}{n} \Delta^{*} \psi+\frac{\varepsilon}{n} I I^{\prime}+R V_{\phi}=R^{2} \omega(\psi), \\
& -\frac{n V_{\phi}^{2}}{R} \nabla R=-\frac{\Delta^{*} \psi}{R^{2}} \nabla \psi-\frac{I I^{\prime}}{R^{2}} \nabla \psi-\nabla p,
\end{aligned}
$$

respectively, where $\omega(\psi)$ is the second surface quantity, and the prime indicates the derivative with respect to $\psi$. The flow effect (the left-hand side of (10)) emerges due to the toroidicity, as we shall see in the next subsection. The force balance equation (10) shows that the flow and the magnetic field are coupled, and that the equilibrium deviates from the static force balance between the Lorentz force and the pressure gradient.

\subsection{Hall MHD equilibrium in a straight cylinder}

To show the importance of the toroidicity, we briefly describe the equilibrium in a straight cylinder, which represents a toroidal geometry with a large aspect ratio. Then (4) yields $\boldsymbol{B}(x, y)=\nabla \psi(x, y) \times \nabla z+B_{z}(x, y) \nabla z$, where $x$ $y-z$ are the standard Cartesian coordinates, and $B_{z}$ is the "toroidal" component of the magnetic field. The assumption of pure toroidal flow leads to $\boldsymbol{V}(x, y)=V_{z}(x, y) \nabla z$, where $V_{z}$ is the toroidal component of the flow velocity.

Substitution of the above expressions into (2) yields

$0=-\Delta \psi \nabla \psi-\left[\left(\nabla \psi \times \nabla B_{z}\right) \cdot \nabla z\right] \nabla z-B_{z} \nabla B_{z}-\nabla p$,

where $\Delta=\partial_{x}^{2}+\partial_{y}^{2}$ is the Laplacian. Equation (11) indicates that the flow velocity and the magnetic field are totally decoupled (cf. (10)), and that the magnetic field is determined by the poloidal flux of current density and the pressure as in the case of static equilibrium, assigning $B_{z}=B_{z}(\psi)$ and $p=p(\psi)$. The resultant GS equation $-\Delta \psi=B_{z} B_{z}^{\prime}+p^{\prime}$ is identical to the static one.

The toroidal flow is determined from (3) as $V_{z}=$ $\Omega(\psi)+\varepsilon p^{\prime} / n$, where $\Omega(\psi)$ is a surface quantity. Since the toroidal flow of the ideal MHD equilibrium in a straight cylinder is written as $V_{z}=\Omega(\psi)$ [12], the term with coefficient $\varepsilon$ is a modification to the ideal MHD flow. Therefore, we conclude that in a straight cylinder, the Hall effect plays a minor role of slightly modifying the ideal MHD flow, and that the toroidicity is indispensable for the significant action of the Hall effect.

\section{Two Types of Equilibria Produced by the Hall Effect}

Using (9), we eliminate the $-R^{-2}\left(\Delta^{*} \psi+I I^{\prime}\right) \nabla \psi$ term in (10), and obtain the force balance equation of the ion fluid,

$$
-\frac{\varepsilon n V_{\phi}^{2}}{R} \nabla R=\frac{n V_{\phi}}{R} \nabla \psi-n \omega \nabla \psi-\varepsilon \nabla p .
$$

Note that the left-hand side of (12) emerges due to the Hall effect and the toroidicity. The toroidal flow $V_{\phi}$, the pressure $p$, and the density $n$ should satisfy (12) and the equation of state.

We assume that the pressure and toroidal flow profiles are in the following forms,

$$
p=p(\psi, R), \quad V_{\phi}=R^{\alpha} f(\psi),
$$


where $\alpha \neq 0$ is an undetermined real number, and $f(\psi)$ is an undermined surface quantity. Substituting (13) into (12) yields

$$
\begin{aligned}
& \varepsilon \frac{\partial p}{\partial \psi}=n R^{\alpha-1} f-n \omega, \\
& \frac{\partial p}{\partial R}=n R^{2 \alpha-1} f^{2} .
\end{aligned}
$$

As is mentioned in Sec. 2, we need the equation of state to close the system. In this paper, we assume the isothermal magnetic surfaces, i.e.,

$$
p(\psi, R)=n(\psi, R) T(\psi),
$$

where $T$ is the temperature which is constant on each magnetic surface due to the high thermal conductivity along magnetic field lines. Then, equation (15) can be integrated as

$$
n(\psi, R)=n_{0}(\psi) \exp \left(\frac{f^{2}}{2 \alpha T} R^{2 \alpha}\right) .
$$

Then (14) reads

$$
\begin{aligned}
& \omega(\psi)+\varepsilon T(\psi) \frac{n_{0}^{\prime}}{n_{0}}+\varepsilon T^{\prime} \\
& \quad+f(\psi)\left[\frac{\varepsilon}{2 \alpha} R^{2 \alpha}\left(2 f^{\prime}-f \frac{T^{\prime}}{T}\right)-R^{\alpha-1}\right]=0 .
\end{aligned}
$$

Since the first three terms of the left-hand side of (18) depend on only $\psi$, we need to restrict the surface quantities $\omega(\psi), T(\psi)$, and $n_{0}(\psi)$ and the unknown $\alpha$ and $f(\psi)$ such as

$$
\begin{aligned}
& \omega(\psi)+\varepsilon T(\psi) \frac{n_{0}^{\prime}}{n_{0}}+\varepsilon T^{\prime}+C_{1} f(\psi)=0, \\
& \frac{\varepsilon C_{2}}{2 \alpha} R^{2 \alpha}-R^{\alpha-1}=C_{1}, \\
& 2 f^{\prime}-f \frac{T^{\prime}}{T}=C_{2},
\end{aligned}
$$

where $C_{1}$ and $C_{2}$ are some constants. In the remainder of this section, we investigate (19)-(21) to show that there exist two types of equilibria.

\subsection{MHD-type equilibrium}

Firstly, we consider the case with $C_{2}=0$, which turns out to give an MHD-type equilibrium that satisfies the ideal MHD model. In this case, from (20) and (21), we get $-R^{\alpha-1}=C_{1}$ and $2 f^{\prime}-f T^{\prime} / T=0$, which yields $\alpha=1$, $C_{1}=-1$, and $f(\psi)=C_{3} \sqrt{T(\psi)}$, where $C_{3}$ is the integration constant.

From (13), the flow is written as

$$
V_{\phi}=R \times C_{3} \sqrt{T(\psi)},
$$

which is similar to the rigid rotation with angular frequency $C_{3} \sqrt{T(\psi)}$ which is a surface function. Equation (19) indicates $C_{3} \sqrt{T(\psi)}=\omega(\psi)+\varepsilon\left[T n_{0}^{\prime} / n_{0}(\psi)+T^{\prime}\right]$, which implies $V_{\phi}=R \omega(\psi)+O(\varepsilon)$. From (17), the density is calculated as

$$
\begin{aligned}
n(\psi, R) & =n_{0}(\psi) \exp \left(\frac{C_{3}^{2}}{2} R^{2}\right) \\
& =n_{0} \exp \left\{\frac{1}{2 T}\left[\omega+\varepsilon\left(T \frac{n_{0}^{\prime}}{n}+T^{\prime}\right)\right]^{2} R^{2}\right\} .
\end{aligned}
$$

Using (9) and (19), we can derive the generalized GS equation as follows,

$$
\begin{aligned}
-\Delta^{*} \psi & =I I^{\prime}+R^{2}\left(n_{0}^{\prime} T+n_{0} T^{\prime}\right) \exp \left(\frac{C_{3}^{2}}{2} R^{2}\right) \\
& =I I^{\prime}+R^{2} \frac{\partial p}{\partial \psi}
\end{aligned}
$$

We can see that the toroidal flow (22), the density (23), and the generalized GS equation (24) derived in this subsection are modified versions of the ideal MHD equilibrium (see Ref. [9] for the ideal MHD case).

\subsection{New-type equilibrium}

We move to the case with $C_{2} \neq 0$, and show that a new-type equilibrium exists. Equation (21) can be solved as $f(\psi)=\sqrt{T(\psi)}\left[C_{4}+C_{2} \int^{\psi} T^{-1 / 2} \mathrm{~d} \psi / 2\right]$, where $C_{4}$ is the integration constant. Equation (20) yields $\alpha=-1, C_{1}=0$, and $C_{2}=-2 / \varepsilon$.

From (13), the flow reads

$$
V_{\phi}=\frac{\sqrt{T(\psi)}}{R}\left[C_{4}-\frac{1}{\varepsilon} \int^{\psi} \frac{\mathrm{d} \psi^{\prime}}{\sqrt{T\left(\psi^{\prime}\right)}}\right],
$$

which is in inverse proportional to $R$, in contrast to the MHD type whose flow is proportional to $R$ [see (22)]. The density derived from (17) reads

$$
\begin{array}{r}
n(\psi, R)=n_{0}(\psi) \exp \left\{-\frac{1}{2 R^{2}}\left[C_{4}\right.\right. \\
\left.\left.-\frac{1}{\varepsilon} \int^{\psi} \frac{\mathrm{d} \psi^{\prime}}{\sqrt{T\left(\psi^{\prime}\right)}}\right]^{2}\right\} .
\end{array}
$$

The generalized GS equation of the new-type equilibrium is derived from (9) and (19) as

$$
\begin{aligned}
-\Delta^{*} \psi=I I^{\prime} & +\left\{\frac{n_{0}(\psi) \sqrt{T(\psi)}}{\varepsilon}\left[C_{4}-\frac{1}{\varepsilon} \int^{\psi} \frac{\mathrm{d} \psi^{\prime}}{\sqrt{T(\psi)}}\right]\right. \\
& \left.+R^{2}\left(n_{0}^{\prime} T+n_{0} T^{\prime}\right)\right\} \\
& \times \exp \left\{-\frac{1}{2 R^{2}}\left[C_{4}-\frac{1}{\varepsilon} \int^{\psi} \frac{\mathrm{d} \psi^{\prime}}{\sqrt{T\left(\psi^{\prime}\right)}}\right]^{2}\right\},
\end{aligned}
$$

which is totally different from the counterpart of the MHD type (cf. (24)). This type of equilibrium does not satisfy the ideal MHD, thus is not derivable from the MHD model. Note that this new-type equilibrium appears due to the coupling of the flow with the Hall and toroidal effects. 


\section{Numerical Analysis of the Two Types of Equilibria}

\subsection{Setting of the problem: application to the RT-1 device}

The generalized GS equations of the two types of equilibria derived in the previous section ((24) and (27)) are numerically solved as boundary value problems to simulate the equilibria in the RT-1 device. In this paper, we confine our attention to how the flow affects the equilibrium configurations.

We set the calculation system as follows. The major and minor radii of the system are $\sim 0.55 \mathrm{~m}$ and $\sim 0.45 \mathrm{~m}$, and those of the levitated ring are $\sim 0.3 \mathrm{~m}$ and $\sim 0.1 \mathrm{~m}$, respectively. We assume $\psi>0$ in the calculation region. The schematic view of the calculation system is shown in Fig. 1.

The expected parameters are $B \sim 0.1 \mathrm{~T}$ near the levitated ring, and $n \sim 8.4 \times 10^{17} \mathrm{~m}^{-3}$ (cut-off density generated by $8.2 \mathrm{GHz}$ Klystron). The expected temperature is not known, and we assume $T \sim 100 \mathrm{eV}$. Table 1 shows the parameters for the RT-1 device, implying that the Hall effect becomes prominent in this system.

We set the surface quantities as follows:

$$
I(\psi)=\alpha_{I} \psi^{2}, \quad T(\psi)=\alpha_{T} \psi^{2}, \quad n_{0}(\psi)=\alpha_{n} \psi^{2},
$$

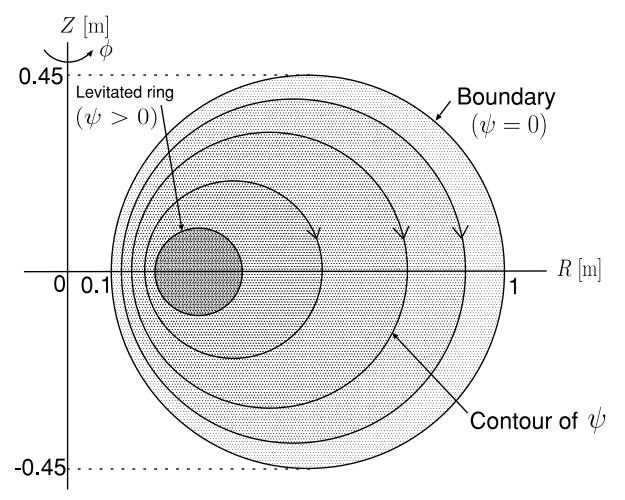

Fig. 1 Schematic view of the calculation system simulating the RT-1 device. The boundary is constituted of the levitated ring producing a dipolar magnetic field and the boundary. In the calculation region, it is assumed that the flux function is positive $(\psi>0)$. where $\alpha_{I}, \alpha_{T}$, and $\alpha_{n}$ are some constants ( $\alpha_{T}$ and $\alpha_{n}$ should be positive). Since the magnetic configuration is almost dipolar in the RT- 1 device, $\left|\alpha_{I}\right| \ll 1$ is required. The second and third equations of (28) imply that the temperature is high and the density without flow is large near the levitated ring where $\psi$ is large. We define the volume-averaged value of a quantity $A$ by $\langle A\rangle=V^{-1} \int A \mathrm{~d} v$, where $V$ is the plasma volume, and $\mathrm{d} v$ is the volume increment. In the present calculation, for simplicity, we fix the volumeaveraged toroidal magnetic field as $\left\langle B_{\phi}\right\rangle=10^{-2} \mathrm{~T}$ to determine the parameter $\alpha_{I}$, and determine the parameters $\alpha_{T}$, and $\alpha_{n}$ by assuming that the volume-averaged values of the temperature and the density become $\langle T\rangle=100 \mathrm{eV}$, and $\langle n\rangle=8.4 \times 10^{16} \mathrm{~m}^{-3}$, respectively.

We need $C_{3}$ and $C_{4}$ for the MHD-type and newtype equilibria, respectively, which are associated with the toroidal flows (see (22) and (25)). Instead of varying these parameters, we vary the volume-averaged toroidal flow $\left\langle V_{\phi}\right\rangle$ to compare both types on equal footing.

In what follows, varying $\left\langle V_{\phi}\right\rangle$ in the range $0 \leq\left\langle V_{\phi}\right\rangle \leq$ 0.08 (in this range, the reasonable solution of the MHDtype equilibrium exists), we observe the behavior of the solutions.

\subsection{Numerical solutions of the MHD-type equilibrium}

We analyze the MHD-type equilibrium by solving (24) for various $\left\langle V_{\phi}\right\rangle$. The contour of the flux function $\psi$ is shown in Fig. 2 (for $\left\langle V_{\phi}\right\rangle=0.06$ ), which indicates the dipolar magnetic field, which is a typical configuration obtained in the present calculation.

Figure 3 shows the profiles of the density (calculated from (23)) and the local beta in the equatorial plane $(Z=0)$ for various volume-averaged toroidal flows, $\left\langle V_{\phi}\right\rangle=0.0$, 0.06, 0.08. From Fig. 3, we observe that the peak of the density (resultantly, the local beta value) shifts outward as $\left\langle V_{\phi}\right\rangle$ increases. The shifts are attributed to the effect of centrifugal force, as is shown in (22) and (23). The maximum of the local beta value is almost $3 \%$ for various $\left\langle V_{\phi}\right\rangle$.

If we increase $\left\langle V_{\phi}\right\rangle$ further, the density peak hits the boundary, and we cannot obtain feasible equilibrium solutions.

Table 1 Calculation parameters for the RT- 1 device

\begin{tabular}{|l|l|l|}
\hline Parameter & Value & Remarks \\
\hline scale length $L_{0}$ & $0.5 \mathrm{~m}$ & minor radius \\
representative density $n_{0}$ & $8.4 \times 10^{17} \mathrm{~m}^{-3}$ & cut-off density of $8.2 \mathrm{GHz}$ Klystron \\
representative magnetic field $B_{0}$ & $0.1 \mathrm{~T}$ & $B$ near the levitated ring \\
Alfvén speed $V_{\mathrm{A}}$ & $2.38 \times 10^{6} \mathrm{~m} / \mathrm{s}$ & derived from $n_{0}$ and $B_{0}$ \\
twice the magnetic pressure $B_{0}^{2} / \mu_{0}$ & $7.96 \times 10^{3} \mathrm{~J} / \mathrm{m}^{3}$ & derived from $B_{0}$ \\
ion skin depth $\delta_{\mathrm{i}}$ & $0.248 \mathrm{~m}$ & derived from $n_{0}$ \\
Hall parameter $\varepsilon$ & 0.496 & derived from $L_{0}$ and $\delta_{\mathrm{i}}$ \\
\hline
\end{tabular}




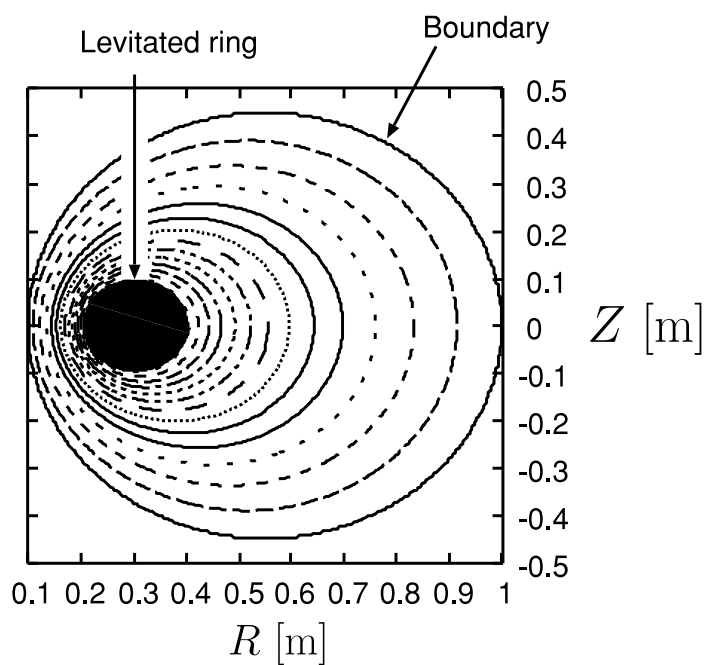

Fig. 2 Contour of flux function $\psi$ of the MHD-type equilibrium for $\left\langle V_{\phi}\right\rangle=0.06\left(\left\langle V_{\phi}\right\rangle\right.$ is the volume-averaged toroidal flow), which indicates the dipolar magnetic configuration.
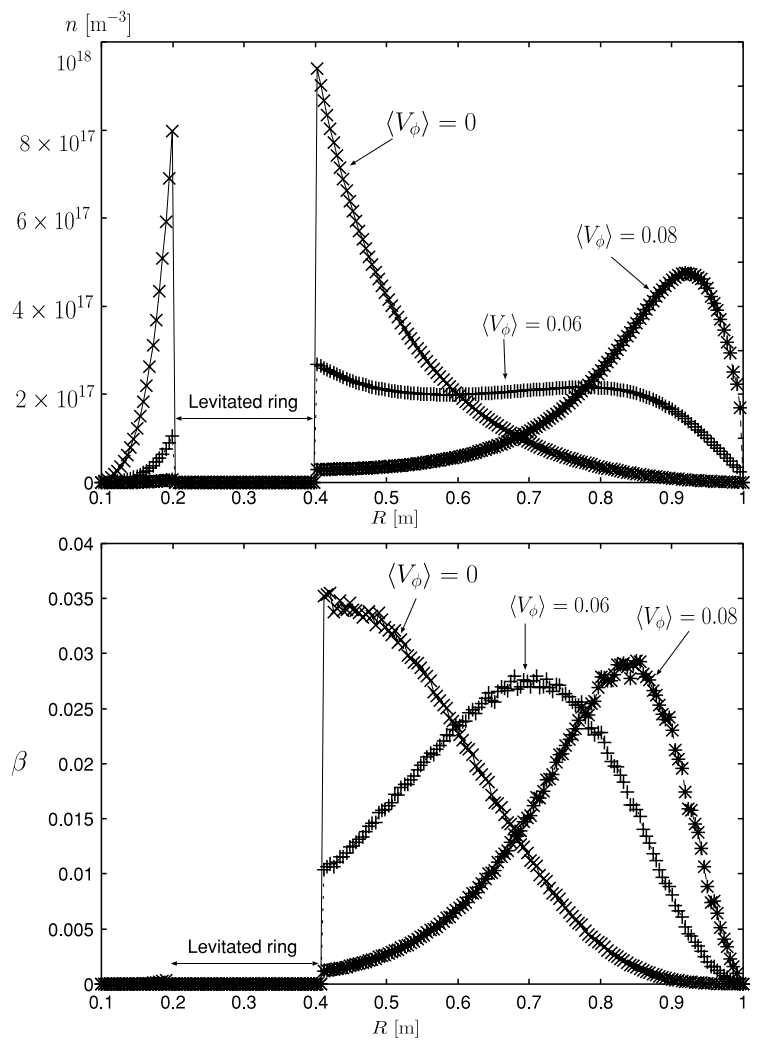

Fig. 3 Behavior of the solution of the MHD-type equilibrium in the equatorial plane $(Z=0)$ with different volumeaveraged toroidal flows, $\left\langle V_{\phi}\right\rangle=0.0,0.06,0.08$. The top panel shows the density profile, and the bottom one shows the local beta value. As $\left\langle V_{\phi}\right\rangle$ increases, the peaks of both quantities shift outward due to the centrifugal effect.

\subsection{Numerical solutions of the new-type equilibrium}

The solutions of the new type, obtained by solving (27), have magnetic surfaces similar to those of the MHD type (see Fig. 2). However, as is clear from (25) and (26), the profiles of the toroidal flow and the density are totally different from those of the MHD type, which brings about qualitatively different behavior of the solution.

In Fig. 4, we show the density and the local beta value profiles in the equatorial plane for the same volumeaveraged toroidal flows as the MHD type. Figure 4 indicates that both peaks shift slightly inward as $\left\langle V_{\phi}\right\rangle$ increases, which is in contrast to the MHD type. The plasma inside the levitated ring in the top panel of Fig. 4 stems from the hollow distribution enclosing the levitated ring, as is shown in Fig. 5 (for $\left\langle V_{\phi}\right\rangle=0.08$ ). The numerical analysis shows that in the new-type equilibrium, the density (and the beta value) localizes in a narrower region, the beta value has a larger amplitude (about 10\%), and the shifts due to the flow effect are smaller compared with the MHDtype solutions.
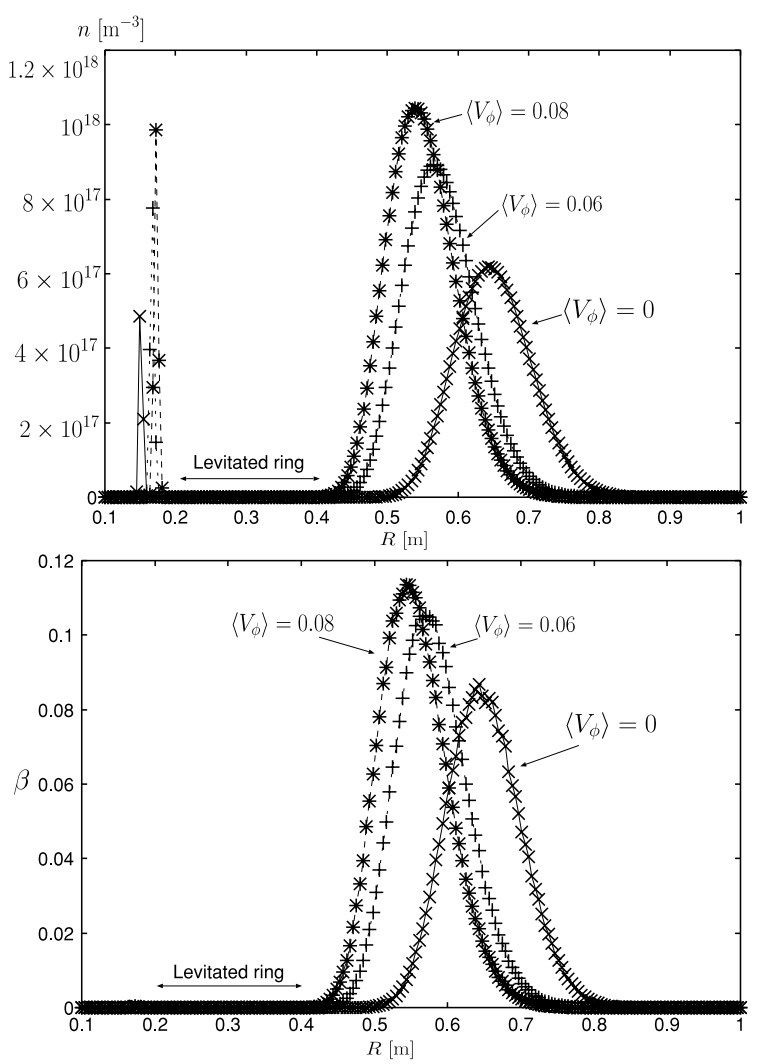

Fig. 4 Behavior of the solution of the new-type equilibrium in the equatorial plane $(Z=0)$ with different volumeaveraged toroidal flows, $\left\langle V_{\phi}\right\rangle=0,0.06,0.08$. The top panel shows the density profile, and the bottom one shows the local beta value. The peaks of both quantities shift inward as $\left\langle V_{\phi}\right\rangle$ increases. Both quantities localize in a narrow region. 


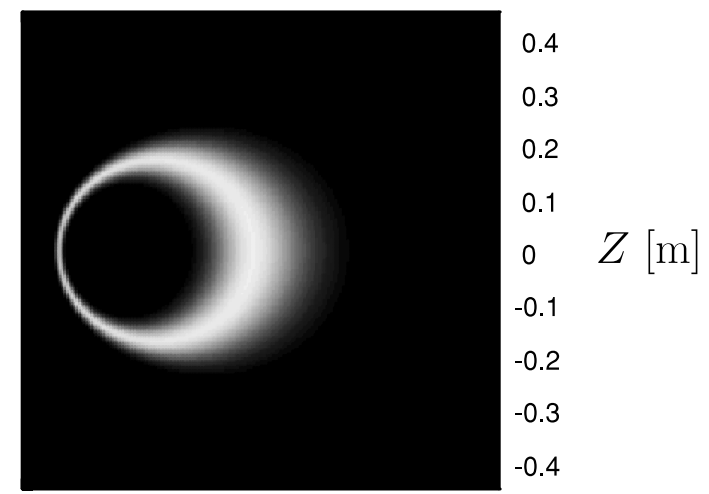

$\begin{array}{llllllllll}0.1 & 0.2 & 0.3 & 0.4 & 0.5 & 0.6 & 0.7 & 0.8 & 0.9 & 1\end{array}$ $R[\mathrm{~m}]$

Fig. 5 Two-dimensional structure of the solution of the newtype equilibrium with $\left\langle V_{\phi}\right\rangle=0.08$. The gray-scale contour represents the density. The plasma encloses the levitated coil, making a hollow profile.

\section{Summary}

We have shown that the axisymmetric MHD equilibrium with toroidal flow has a new-type equilibrium produced due to the coexistence of the Hall effect and toroidicity. The density and flow profiles of this new-type equilibrium are significantly different from those of the conventional MHD type; thus, it is expected that their stability and confinement properties are qualitatively different.

The generalized GS equations of both types have been numerically solved in toroidal geometries to simulate the equilibria in the RT-1 device. As the volume-averaged toroidal flow increases, the peaks of the density and local beta of the MHD-type shift outward, which is attributed to the centrifugal effect induced by the flow similar to the rigid rotation (cf. (22)). In contrast, the density and local beta peaks of the new-type equilibrium shift inward as the toroidal flow is increased. This contrary behavior may stem from the fact that the flow profile, which is proportional to $R^{-1}$ (see (25)), is very different from the MHD type, and resultantly the dependence of the density on $R$ is also different (compare (23) with (26)). In the new-type equilibrium, the density and the beta value localize in a narrower region, the maximum of the local beta reaches a higher value $\sim 10 \%$, and the shifts due to the flow effect are smaller than the MHD type (see Figs. 3 and 4).

We end this paper by making two remarks. First, we note that the equilibria obtained in this paper have no poloidal flow, which makes the analysis mathematically tractable. In the presence of poloidal flow, problems of hyperbolicity [13] may occur, which indicates that the equilibrium cannot be obtained as a boundary value problem. Thus, obtaining equilibria with poloidal flows is a very tough problem. Secondly, we note that the theory presented in this paper does not clarify which type of equilibrium is experimentally realized, nor the condi- tion necessary to achieve it, which are beyond the scope of the present study. To resolve these problems, a stability analysis should be performed, which is a very challenging problem because of the existence of the flow and the twodimensionality, and will be reported elsewhere.

This work was supported by Grant-in-Aid for Scientific Research from the Japanese Ministry of Education, Culture, Sports, Science and Technology, No. 14102033.

\section{Appendix: Computational Method of Solving the Generalized Grad- Shafranov Equation}

In this appendix, we shortly delineate the computational technique used in this paper to solve the generalized GS equations ((24) and (27)) with Dirichlet boundary conditions. The problem reads $-\Delta^{*} \psi=F(\psi)$ where $F(\psi)$ is some nonlinear function and $\psi=\psi_{\mathrm{b}}(R, Z)$ on the boundary. Since the equation is nonlinear, the iteration method is invoked, which leads to

$$
-\Delta^{*} \psi^{n+1}=F^{n}(R, Z):=F\left(\psi^{n}\right),
$$

where $n$ is the iteration step. The numerical solution $\psi^{n+1}$ is obtained when $\max \left[\left|\psi^{n+1}-\psi^{n}\right| / \max \left(\psi^{n}\right)\right]<\epsilon$ is achieved, where $\epsilon$ is a sufficiently small number. We rewrite the above equation in the form of integral equation as

$$
\psi^{n+1}=\left(-\Delta^{*}\right)^{-1} F^{n}(R, Z),
$$

where the inverse operator $\left(-\Delta^{*}\right)^{-1}$ can be calculated by

$$
\begin{aligned}
& \left(-\Delta^{*}\right)^{-1} F^{n}(R, Z) \\
& =\int G\left(R, Z, R^{\prime}, Z^{\prime}\right) \frac{F^{n}\left(R^{\prime}, Z^{\prime}\right)}{R^{\prime}} \mathrm{d} R^{\prime} \mathrm{d} Z^{\prime},
\end{aligned}
$$

where

$$
\begin{aligned}
& G\left(R, Z, R^{\prime}, Z^{\prime}\right):=\frac{1}{\pi \kappa} \sqrt{R R^{\prime}}\left[\left(1-\frac{\kappa^{2}}{2}\right) K(\kappa)-E(\kappa)\right], \\
& \kappa^{2}:=\frac{4 R R^{\prime}}{\left(R+R^{\prime}\right)^{2}+\left(Z-Z^{\prime}\right)^{2}} .
\end{aligned}
$$

Here $G\left(R, Z, R^{\prime}, Z^{\prime}\right)$ denotes the Green function, and $K(\kappa)$ $(E(\kappa))$ is the complete elliptic integral of the first (second) kind. Note that $F^{n}\left(R^{\prime}, Z^{\prime}\right) / R^{\prime}$ indicates the toroidal current density. At the beginning of the iteration, we drive uniform toroidal current in the plasma region $\left(F^{0}=\right.$ constant $)$.

The Dirichlet boundary condition is treated as follows. We allocate many points representing the external current $I_{\mathrm{ex}}^{(j)}$ and the boundary flux $\psi_{\mathrm{b}}^{(i)}$ as is shown in Fig. 6. First we construct a matrix that relates the external current with the boundary flux. We substitute 1 into external current $I_{\mathrm{ex}}^{(j)}$, calculate the $i$-th boundary flux by $\left(-\Delta^{*}\right)^{-1} I_{\mathrm{ex}}^{(j)}$, and define the matrix $A_{i j}$ such that $A_{i j}$ corresponds to the flux at the $i$-th boundary produced by the $j$-th external current (fixed to unity). Next we calculate the $i$-th boundary flux produced by the plasma current as $\psi_{\mathrm{p}}^{(i)}=\left(-\Delta^{*}\right)^{-1} I_{\mathrm{p}}$, and 


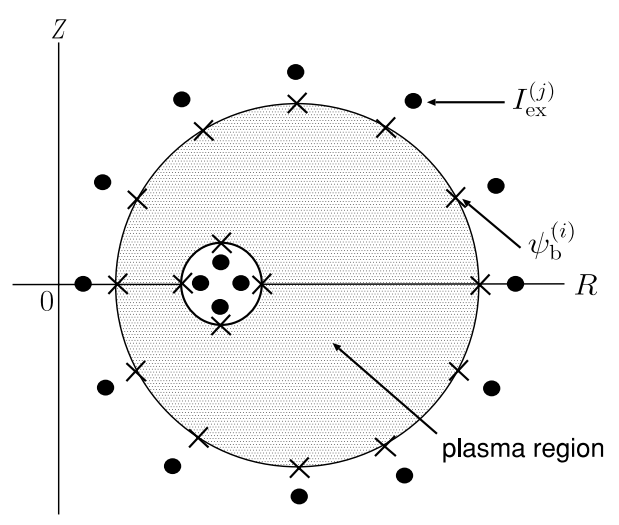

Fig. 6 Explanation drawing for the calculation method. The black circles denote the external current $I_{\mathrm{ex}}^{(j)}$, and the xmarks represent the points for the boundary condition $\psi_{\mathrm{b}}^{(i)}$, both of which are located in the levitated ring and the boundary (reduced points are shown for illustrative purpose). The plasma exists in the shaded region.

define $\hat{\psi}_{\mathrm{b}}^{(i)}:=\psi_{\mathrm{p}}^{(i)}-\psi_{0}^{(i)}$ where $\psi_{0}^{(i)}$ is the given value at $i$-th boundary. Using the inverse matrix $A_{i j}^{-1}$, we set the external current by computing $I_{\mathrm{ex}}^{(j)}=-\sum_{i} A_{i j}^{-1} \hat{\psi}_{\mathrm{b}}^{(i)}$. Then, since the boundary flux produced by the external current is calculated by $\psi_{\mathrm{ex}}^{(i)}=\sum_{j} A_{i j} I_{\mathrm{ex}}^{(j)}$, the flux of the $i$-th boundary reads $\psi_{\mathrm{b}}^{(i)}=\psi_{\mathrm{p}}^{(i)}+\psi_{\mathrm{ex}}^{(i)}=\psi_{0}^{(i)}$, which satisfies the boundary condition.
[1] Z. Yoshida, Y. Ogawa, J. Morikawa, S. Watanabe, Y. Yano, S. Mizumaki, T. Tosaka, Y. Ohtani, A. Hayakawa and M. Shibui, Plasma Fusion Res. 1, 008 (2006).

[2] H. Saitoh, Z. Yoshida, C. Nakashima, H. Himura, J. Morikawa and M. Fukao, Phys. Rev. Lett. 92, 255005 (2004).

[3] H. Saitoh, Z. Yoshida, H. Himura, J. Morikawa and M. Fukao, Phys. Plasmas 11, 3331 (2004).

[4] J. Shiraishi, S. Ohsaki and Z. Yoshida, Phys. Plasmas 12, 092901 (2005).

[5] J. Kesner, L. Bromberg, D. Garnier and M.E. Mauel, in 17th IAEA Fusion Energy Conference, Paper No.IAEAF1-CN-69-ICP/09 Yokohama, Japan (International Atomic Energy Agency, Vienna, 1998).

[6] S.M. Mahajan and Z. Yoshida, Phys. Rev. Lett. 81, 4863 (1998).

[7] Z. Yoshida and S.M. Mahajan, Phys. Rev. Lett. 88, 095001 (2002).

[8] S.M. Mahajan and Z. Yoshida, Phys. Plasmas 7, 635 (2000).

[9] M. Furukawa, Y. Nakamura, S. Hamaguchi and M. Wakatani, J. Plasma Fusion Res. 76, 937 (2000).

[10] P.J. Catto and S.I. Krasheninnikov, Phys. Lett. A 258, 153 (1999).

[11] S.I. Krasheninnikov, P.J. Catto and R.D. Hazeltine, Phys. Plasmas 7, 1831 (2000).

[12] G.N. Throumoulopoulos and H. Tasso, Phys. Plasmas 4, 1492 (1997).

[13] J.P. Goedbloed, Phys. Plasmas 11, L81 (2004). 